\title{
Selection and Fusion of Similarity Measure Based Classifiers Using Support Vector Machines
}

\author{
Mohammad T. Sadeghi ${ }^{1}$, Masoumeh Samiei ${ }^{1}$, and Josef Kittler ${ }^{2}$ \\ ${ }^{1}$ Signal Processing Research Laboratory \\ Department of Electrical and Computer Engineering \\ Yazd University, Yazd, Iran \\ 2 Centre for Vision, Speech and Signal Processing \\ School of Electronics and Physical Sciences \\ University of Surrey, Guildford GU2 7XH, UK \\ \{M.Sadeghi, J.Kittler\}@surrey.ac.uk
}

\begin{abstract}
In this paper, we address the problem of selecting and fusing similarity measures based classifiers in LDA face space. The performance of a face verification system in an LDA feature space using different similarity measure based classifiers is experimentally studied first. The study is performed for both manually and automatically registered face images. A sequential search approach which is in principle similar to the "plus L and take away R" algorithm is then applied in order to find an optimum subset of the adopted classifiers. The selected classifiers are combined using the SVM classifier. We show that although, individually, one of the adopted scoring functions, the Gradient Direction distance outperforms the other metrics, by fusing different similarity measures using the proposed method, the resulting decision making scheme improves the performance of the system in different conditions.
\end{abstract}

\section{Introduction}

In spite of the rapid advances in machine learning, in many pattern recognition problems the decision making is based on simple concepts such as distance from or similarity to some reference patterns. This type of approach is particularly relevant when the number of training samples available to model a class of objects is very limited. Examples of such situations include content based retrieval from image or video databases, where the query image is the only sample at our disposal to define the object model, or biometrics where only one or a few biometric traits can be acquired during subject enrolment to create a reference template. In biometric identity verification, a similarity function measures the degree of similarity of an unknown pattern to the claimed identity template. If the degree exceeds a prespecified threshold, the unknown pattern is accepted to be the same as the claimed identity. Otherwise it is rejected.

The similarity score is computed in a suitable feature space. Commonly, similarity would be quantified in terms of a distance function, on the grounds that 
similar patterns will lie physically close to each other. Thus smaller the distance, the greater the similarity of two entities.

The role of the feature space in similarity measurement is multifold. First of all the feature space is selected so as to maximise the discriminatory information content of the data projected into the feature space and to remove any redundancy. However, additional benefit expected from mapping the original pattern data into a feature space is to simplify the similarity measure deployed for decision making. Linear Discriminant Analysis (LDA) is a powerful feature extraction tool for pattern recognition in general and for face recognition in particular. It was introduced to this application area by Belhumeur in 1996 [1. An important contributing factor in the performance of a face authentication system within the LDA space is the metric used for defining a matching score. Theoretically, Euclidean distance provides an optimal measure in the LDA space. However, it has been demonstrated that it is outperformed by the Normalised Correlation (NC) and Gradient Direction (GD) 12. Also, in [13, the performance of the NC scoring function was compared with the GD metric. The study was performed on the BANCA database 1 using internationally agreed experimental protocols by applying a geometric face registration method based on manually or automatically annotated eyes positions. It was concluded that overall the NC function is less sensitive to miss-registration error but in certain conditions GD metric performs better. However, in [14, it has been further demonstrated that by optimising the GD metric, this metric almost always outperforms the NC metric for both manually and automatically registered data. A variety of other metrics has been adopted as the similarity measure in different pattern recognition applications. Experimental results confirm that, individually other metrics, overall, do not perform as well as the NC and GD metrics in the LDA space for face verification. However, in different conditions, different classifiers can deliver better performance.

In this work we adopted 8 classifiers based on different similarity measures including Euclidean, City-block, Chebyschev, Canberra, Chi-square $\left(\chi^{2}\right)$, NC, GD and Correlation coefficient-based distance. It is well known that a combination of many different classifiers can improve classification accuracy. The main idea behind the current study is to select and fuse the best classifiers in different conditions.

A variety of schemes have been proposed for combining multiple classifiers. Fusion rules can be divided into two main categories: fixed rules such as the sum, minimum, maximum and median rule [6] [7] 17] and trained rules like the weighted averaging of classifiers outputs [16] 11], Support Vector Machines (SVM) 14, bagging and boosting [4. In [8], AdaBoost has been adopted for combining unimodal features extracted from face and speech signals of individuals in multimodal biometrics. Overall, the fixed rules are most often used because of their simplicity and do not requiring to a training data. In such a way, equal weights are used for all the classifiers [6] 3]. However, in different applications it has been demonstrated that trained approaches such as Support

${ }^{1}$ http://www.ee.surrey.ac.uk/banca/ 
Vector Machines (SVMs) have the potential to outperform the simple fusion rules, especially when a large enough training data is available.

Our approach to the classifier combination is at the decision-level. The individual classifiers are based on different similarity measures and each classifier outputs a score. The scores are treated as features and a second level classifier such as support vector classifiers, neural networks, Parzen classifier, etc., is constructed by training on these scores. Therefore, it is pertinent to ask which classifiers provide complementary information and how the expert scores should be fused to achieve the best possible performance of the face verification system. In 13. the fusion problem was solved by selecting the best classifier or a group of classifiers dynamically with the help of a gating function learnt for each similarity measures. In the present study we formulate the similarity measures fusion problem as a feature selection problem. The similarity measures are selected using a sequential search approach, the Plus L and Take Away R algorithm [2].

An important contributing factor in the selection algorithm is the "fusion rule" used for combining the similarity measure based classifiers. The experimental results in 14 confirm that, by fusing experts employing diverse similarity measures using the SVM classifier, the performance of the system is superior to that of any single similarity measure. It also improves compared to classical non trainable fusion strategies.

The main aim of this paper is to study the performance of the proposed similarity measure selection algorithm using the SVM classifiers. Surprisingly good results are obtained using the proposed method. The paper is organised as follows. In the next section the adopted scoring functions are introduced. The proposed method of classifier selection is described in Section 3 . A description of the experimental design including the face database used in the study, the experimental protocols and the experimental setup are given in Section 4] The experimental results using the adopted scoring functions and the fusion results are presented and discussed in Section 5. Finally a summary of the main findings and conclusions can be found in Section 6 .

\section{Similarity Functions}

In a similarity measure based face verification system, a matching scheme measures the similarity or distance of the test sample, $\mathbf{x}$ to the template of the claimed identity, $\boldsymbol{\mu}_{i}$. Note that $\mathbf{x}$ and $\boldsymbol{\mu}_{i}$ are the projections of the test sample and class mean into the feature space respectively. The general form of a group of similarity measures which is called Minkowski Distance or power norm metrics $\left(L_{p}\right)$ is defined as:

$$
s_{M}=\left|\sum_{j=1}^{m}\left(\boldsymbol{\mu}_{i j}-\mathbf{x}_{j}\right)^{p}\right|^{1 / p}
$$

where $\mathrm{m}$ is the dimensionality.

The most commonly used similarity measures, Manhattan or City-block metric, Euclidean Distance (ED) and Chebyschev Distance are derived from the 
above definition considering $p=1, p=2$ and $p \rightarrow \infty$ respectively , i.e. $L_{1}, L_{2}$ and $L_{\infty}$ metrics:

$$
s_{C i t y}=\sum_{j=1}^{m}\left|\boldsymbol{\mu}_{i j}-\mathbf{x}_{j}\right|, \quad s_{E D}=\sqrt{\left(\mathbf{x}-\boldsymbol{\mu}_{i}\right)^{T}\left(\mathbf{x}-\boldsymbol{\mu}_{i}\right)}, \quad s_{C h e b}=\max _{j}\left|\boldsymbol{\mu}_{i j}-\mathbf{x}_{j}\right|
$$

The Canberra Distance is also given by

$$
s_{C a n b}=\sum_{j=1}^{m} \frac{\left|\boldsymbol{\mu}_{i j}-\mathbf{x}_{j}\right|}{\left|\boldsymbol{\mu}_{i j}\right|+\left|\mathbf{x}_{j}\right|}
$$

This can be considered as the normalised Manhattan Distance. The Chi-squared $\left(\chi^{2}\right)$ Distance is defined by

$$
s_{\chi^{2}}=\sum_{j=1}^{m} \frac{\left(\boldsymbol{\mu}_{i j}-\mathbf{x}_{j}\right)^{2}}{\left|\boldsymbol{\mu}_{i j}\right|+\left|\mathbf{x}_{j}\right|}
$$

which is basically a relative Euclidean squared distance and is usually meant for non negative variables only.

In different applications, it has been shown that a matching score based on Normalised Correlation (NC) scoring function, defined by Equation 5, is more efficient.

$$
s_{N C}=\frac{\left\|\mathbf{x}^{T} \boldsymbol{\mu}_{i}\right\|}{\sqrt{\mathbf{x}^{T} \mathbf{x} \boldsymbol{\mu}_{i}^{T} \boldsymbol{\mu}_{i}}}
$$

Another similarity measure which is conceptually same as the NC function is Distance based Correlation Coefficients. For more details, the reader is referred to 9 .

The Gradient Direction (GD) metric measures the distance between a probe image and a model in the gradient direction of the aposteriori probability function associated with the hypothesised client identity $i$ 12. A mixture of Gaussian distributions with isotropic covariance matrix has been assumed as the density function representing the anticlass (world population) estimated from the data provided by all the other users $(\forall j \neq i)$. The diagonal elements of the isotropic covariance matrix are assumed to have values related to the magnitude of variation of the image data in the feature space. It was demonstrated that applying GD metric is even more efficient than the $\mathrm{NC}$ function. The matching score is defined as

$$
s_{G D}=\frac{\left\|\left(\mathbf{x}-\boldsymbol{\mu}_{i}\right)^{T} \nabla_{O} P(i \mid \mathbf{x})\right\|}{\left\|\nabla_{O} P(i \mid \mathbf{x})\right\|}
$$

where $\nabla_{O} P(i \mid \mathbf{x})$ refers to the optimal gradient direction. For the isotropic structure of the covariance matrix, i.e. $\boldsymbol{\Sigma}=\sigma \mathbf{I}$, the optimal direction would be

$$
\nabla_{I} P(i \mid \mathbf{x})=\sum_{j \neq i, j=1}^{m} p(\mathbf{x} \mid j)\left(\boldsymbol{\mu}_{j}-\boldsymbol{\mu}_{i}\right)
$$


Note that the magnitude of $\sigma$ will affect the gradient direction through the values of density $p(\mathbf{x} \mid j)$.

\section{Similarity Measure Based Classifiers Selection}

One of the very promising research directions in the field of pattern recognition and computer vision is classifier fusion. It has been recognised that the classical approach to designing a pattern recognition system which focuses on finding the best classifier has a serious drawback. Any complementary discriminatory information that other classifiers may capture is not tapped. Multiple expert fusion aims to make use of many different designs to improve the classification performance. In the case considered here, as different metrics span the feature space in different ways, it seems reasonable to expect that a better performance could be obtained by combining the resulting classifiers.

Multiple expert fusion aims to make use of many different designs to improve the classification performance. The approach we adopted for selecting the best set of classifiers is similar in principal to the sequential feature selection methods 10. In this study, the Sequential Forward Selection (SFS) and Plus'L' and Take away ' $R$ ' algorithms were examined for selecting an optimum subset of similarity measures. A trained fusion rule, realised by a Support Vector Machine, is used to combine the scores of the selected similarity based classifiers. The selection procedure keeps adding or taking away features (similarity measures in our case) until the best evaluation performance is achieved. Only the selected similarity measures are then used for decision making in the final multiple classifier system.

A Support Vector Machine is a two-class classifier showing superior performance to other methods in terms of Structural Risk 15. In this study, decision level fusion approach drawing on SVMs has been adopted for combining the similarity measure based classifiers. For the face verification problem, the size of the training set for clients is usually less than that for impostors. In such a case, the class of impostors is represented better. Therefore, it is necessary to shift the optimal hyperplane of the SVM classifier towards the better represented class. In this work, the size of the shift is determined in the evaluation step using the Equal Error Rate criterion.

\section{Experimental Design}

In this section the face verification experiments carried out on images of the BANCA database are described. The BANCA database is briefly introduced first. The main specification of the experimental setup is then presented.

\subsection{BANCA Database}

The BANCA database has been designed in order to test multi-modal identity verification systems deploying different cameras in different scenarios (Controlled, Degraded and Adverse). The database has been recorded in several 
Table 1. The usage of the different sessions in the BANCA experimental protocols

\begin{tabular}{||c|c||c|c|c|c|c|c|c|c|c|c|c|c||}
\hline Name & Symbol & 1 & 2 & 3 & 4 & 5 & 6 & 7 & 8 & 9 & 10 & 11 & 12 \\
\hline Matched Controlled & Mc & TI & CI & CI & CI & & & & & & & & \\
\hline Matched Degraded & Md & & & & & TI & CI & CI & CI & & & & \\
\hline Matched Adverse & Ma & & & & & & & & & TI & CI & CI & CI \\
\hline Unmatched Degraded & Ud & T & & & & I & CI & CI & CI & & & & \\
\hline Unmatched Adverse & Ua & T & & & & & & & & I & CI & CI & CI \\
\hline Pooled test & P & TI & CI & CI & CI & I & CI & CI & CI & I & CI & CI & CI \\
\hline Grand test & G & TI & CI & CI & CI & TI & CI & CI & CI & TI & CI & CI & CI \\
\hline
\end{tabular}

languages in different countries. Our experiments were performed on the English section of the database. Each section contains 52 subjects (26 males and 26 females).

Each subject participated to 12 recording sessions in different conditions and with different cameras. Sessions 1-4 contain data under Controlled conditions whereas sessions 5-8 and 9-12 contain Degraded and Adverse scenarios respectively. In order to create more independent experiments, images in each session have been divided into two groups of 26 subjects (13 males and 13 females). Experiments can be performed on each group separately. In the BANCA protocol, 7 different distinct experimental configurations have been specified. Table 1 describes the usage of the different sessions in each configuration. "T" refers to the client training while "C" and "I" depict client and impostor test sessions respectively.

\subsection{Experimental Setup}

The performance of different decision making methods discussed in Section 2 is experimentally evaluated on the BANCA database using the configurations discussed in the previous section. The evaluation is performed in the LDA space. The original resolution of the image data is $720 \times 576$. The experiments were performed with a relatively low resolution face images, namely $64 \times 49$. The results reported in this article have been obtained by applying a geometric face normalisation based on the eyes positions. The eyes positions were localised either manually or automatically. A fast method of face detection and eyes localisation was used for the automatic localisation of eyes centre [5]. The XM2VTS database 2 was used for calculating the LDA projection matrix.

The thresholds in the decision making system have been determined based on the Equal Error Rate criterion, i.e. by setting the operating point so that the false rejection rate $(\mathrm{FRR})$ is equal to the false acceptance rate (FAR). The thresholds are set either globally $(G T)$ or using the client specific thresholding $(C S T)$ technique 12. In the training sessions of the BANCA database 5 client images per person are available. In the case of global thresholding method, all these images are used for training each client template. The other group data is then used to set the threshold. In the case of the client specific thresholding

${ }^{2}$ http://www.ee.surrey.ac.uk/Research/VSSP/xm2vtsdb/ 
strategy, only two images are used for the template training and the other three along with the other group data are used to determine the thresholds. Moreover, in order to increase the number of data used for training and to take the errors of the geometric normalisation into account, 24 additional face images per each image were generated by perturbing the location of the eyes position around the annotated positions.

In the previous studies 12, it was demonstrated that the Client Specific Thresholding (CST) technique is superior in the matched scenario ( $\mathrm{Mc}, \mathrm{Md}, \mathrm{Ma}$ and G) whereas the Global Thresholding (GT) method gives a better performance on the unmatched protocols. The results reported in the next section using thresholding were acquired using this criterion.

Also, the SVM classifier has been used in order to fuse the selected classifiers.

\section{Experimental Results and Discussion}

Tables 2 contains a summary of the results obtained using the individual scoring function on the evaluation and test sets when manually annotated eyes position were used for the face geometric normalisation. The values in the table indicate the Total Error rates in the Evaluation (TEE) and Test (TET) stages respectively. These results clearly demonstrate that among the adopted metric, individually, the GD metric is the outright winner. In a few cases, the NC results are comparable to the GD one. The performance of the other metrics is much worse. Results of the similar experiments considering automatically registered data demonstrate that in such a condition also the GD function deliver a better or at least comparable performance [14.

In the next step, the problem of combining classifiers derived from different similarity measures using the SVMs was considered. Table 3 contains the combined verification results using manually and automatically registered data. These results demonstrate that a better performance is achieved using the combined method especially on Mc and G protocols.

Table 2. ID verification results obtained using different scoring functions with Global and Client Specific Thresholding techniques for unmatched and matched protocols respectively. TEE: Total Error rate Evaluation and TET: Total Error rate Test.

\begin{tabular}{|c|c|c|c|c|c|c|c|c|c|c|c|c|c|c|}
\hline \multirow{2}{*}{} & \multicolumn{2}{|c|}{ Mc } & \multicolumn{2}{c|}{ Md } & \multicolumn{2}{c|}{ Ma } & \multicolumn{2}{c|}{ Ud } & \multicolumn{2}{c|}{ Ua } & \multicolumn{2}{c|}{ P } & \multicolumn{2}{c|}{ G } \\
\cline { 2 - 16 } & TEE & TET & TEE & TET & TEE & TET & TEE & TET & TEE & TET & TEE & TET & TEE & TET \\
\hline NC & 1.93 & 8.08 & 3.57 & 13.36 & 3.79 & 14.61 & 24.81 & 25.93 & 37.63 & 38.81 & 27.69 & 28.01 & 7.26 & 9.75 \\
\hline GD & 0.60 & 4.87 & 1.77 & 7.18 & 1.55 & 8.03 & 26.09 & 24.74 & 27.5 & 27.40 & 19.56 & 19.64 & 2.43 & 4.12 \\
\hline ED & 7.97 & 25.89 & 17 & 32.34 & 25.06 & 38.62 & 52.37 & 51.15 & 59.26 & 60.42 & 47.12 & 48.22 & 46.33 & 54.3 \\
\hline City & 11.6 & 29.65 & 22.9 & 37.4 & 34.17 & 43.71 & 57.82 & 58.4 & 66.44 & 67.3 & 54.25 & 54.25 & 57.24 & 62.26 \\
\hline Cheb & 8.2 & 31.73 & 16.22 & 39.23 & 16 & 35.86 & 56.44 & 56.3 & 58.94 & 57.41 & 51.56 & 51.85 & 32.54 & 43.79 \\
\hline$\chi^{2}$ & 7.49 & 20.41 & 14.88 & 28.88 & 22.99 & 34.17 & 48.17 & 47.15 & 56.35 & 60.48 & 44.46 & 45.45 & 42.91 & 48.12 \\
\hline Corr & 2.25 & 11.22 & 4.74 & 15.6 & 4.54 & 17.43 & 22.66 & 26.25 & 36.57 & 37.44 & 34.44 & 34.54 & 8.02 & 10.85 \\
\hline Canb & 5 & 13.85 & 8.69 & 20.25 & 12.01 & 24.2 & 34.26 & 33.5 & 51.54 & 52.37 & 26.74 & 27.69 & 22.54 & 24.04 \\
\hline
\end{tabular}




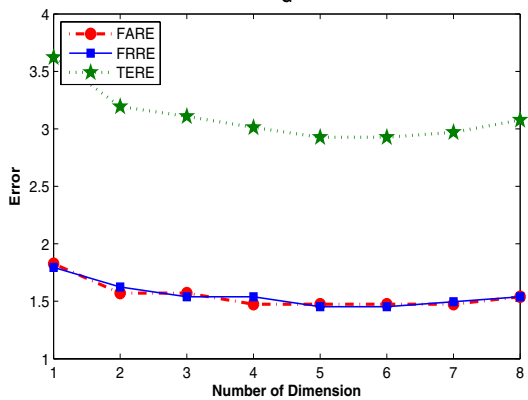

(a) Evaluation (Manual registration)

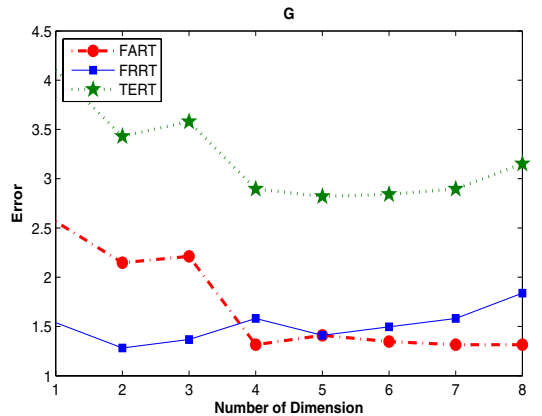

(b) Test (Manual registration)

Fig. 1. ID verification results obtained when a subset of similarity measure based classifiers is selected using the SFS algorithm. SVM has been used for classifier fusion.

Then, the sequential search approaches, SFS and Plus 'L' and Take away 'R' algorithms with $L=2$ and $R=1$ were used for selecting a subset of similarity measure based classifiers. For this purpose, the scores associated to each similarity measure were appropriately normalised first. The normalised scores were then considered as a feature vector. The SVM classifier was finally used for decision making.

Figure 1 shows the resulting error rates for different number of similarity measures considering the G protocol using SFS algorithm. Figures 1 (a) and (b) contain the results considering the evaluation and test data respectively. Please note that based on the SFS algorithm, in each step, an optimum subset of the classifiers is selected considering the performance of the system using the evaluation data. The selected classifiers are then applied to the test data. As the evaluation plots show, when the number of the classifiers increases, the resulting error rate decreases first, then the error rate increases gradually. Moreover, the test results are almost consistent with the evaluation ones. Therefore, it is possible to find an optimum subset of the classifiers. Similar results were obtained using the Plus 'L' and Take away 'R' algorithm.

Table 3. ID verification results considering SVMs for combining classifiers derived from different metrics, manual registration(left) and automatic registration (right)

\begin{tabular}{|c||c|c|c||c|c|c||c|c|c||c|c|c|}
\hline \multicolumn{1}{|c||}{} & \multicolumn{4}{c||}{ Manual Registration } & \multicolumn{4}{c|}{ Automatic Registration } \\
\cline { 2 - 14 } & \multicolumn{3}{|c|}{ Evaluation } & \multicolumn{3}{c||}{ Test } & \multicolumn{3}{c|}{ Evaluation } & \multicolumn{3}{c|}{ Test } \\
\cline { 2 - 6 } & FAR & FRR & TER & FAR & FRR & TER & FAR & FRR & TER & FAR & FRR & TER \\
\hline Mc & 0.96 & 1.03 & 1.99 & 0.86 & 1.54 & 2.4 & 8.17 & 8.2 & 16.37 & 5.96 & 9.36 & 15.32 \\
\hline $\mathrm{Md}$ & 3.75 & 3.85 & 7.6 & 2.98 & 3.97 & 6.95 & 8.36 & 8.46 & 16.82 & 12.79 & 6.28 & 19.07 \\
\hline $\mathrm{Ma}$ & 2.4 & 2.31 & 4.71 & 3.65 & 5.27 & 8.92 & 5.57 & 5.51 & 11.08 & 2.02 & 15.9 & 17.92 \\
\hline $\mathrm{Ud}$ & 9.61 & 9.61 & 19.22 & 10.38 & 13.46 & 23.84 & 13.46 & 13.46 & 26.92 & 15.09 & 15 & 30.09 \\
\hline $\mathrm{Ua}$ & 12.88 & 13.08 & 25.96 & 16.63 & 10.39 & 27.02 & 17.88 & 17.82 & 35.7 & 28.17 & 23.08 & 51.25 \\
\hline $\mathrm{P}$ & 8.87 & 8.85 & 17.72 & 9.29 & 9.06 & 18.35 & 15.64 & 15.6 & 31.24 & 14.33 & 16.62 & 30.95 \\
\hline $\mathrm{G}$ & 1.54 & 1.54 & 3.08 & 1.31 & 1.84 & 3.15 & 7.05 & 7.05 & 14.1 & 7.53 & 8.76 & 16.29 \\
\hline
\end{tabular}


Table 4. ID verification results using the proposed similarity measures selection method considering SVMs for combining classifiers derived from different metrics

\begin{tabular}{|c||c|c||c|c||c||c||c|c|}
\hline \multicolumn{1}{|c||}{} & \multicolumn{3}{c||}{ Manual Registration } & \multicolumn{2}{|c|}{ Automatic Registration } \\
\cline { 2 - 9 } & \multicolumn{2}{|c|}{ SFS } & \multicolumn{2}{c|}{$+2-1$} & \multicolumn{2}{c|}{ SFS } & \multicolumn{2}{|c|}{$+2-1$} \\
\cline { 2 - 9 } & TEE & TET & TEE & TET & TEE & TET & TEE & TET \\
\hline Mc & 1.99 & 2.14 & 1.99 & 2.14 & 16.14 & 23.88 & 16 & 22.17 \\
\hline Md & 7.37 & 6.86 & 7.37 & 6.86 & 16.82 & 19.07 & 16.82 & 19.07 \\
\hline Ma & 4.71 & 8.9 & 4.71 & 8.9 & 11.08 & 17.92 & 11.08 & 17.92 \\
\hline Ud & 18.97 & 21.31 & 18.97 & 21.31 & 26.92 & 30.09 & 26.92 & 30.09 \\
\hline Ua & 25.93 & 26.22 & 25.7 & 26.22 & 35.7 & 51.25 & 35.25 & 40.86 \\
\hline P & 17.44 & 18.29 & 17.32 & 18 & 31.24 & 30.95 & 31.24 & 30.95 \\
\hline G & 2.92 & 2.82 & 2.86 & 3.19 & 13.95 & 14.49 & 13.95 & 14.49 \\
\hline
\end{tabular}

Table 4 contains the fusion results obtained using the SFS and "Plus 2 and take away 1" algorithms considering the manually and automatically registered data. As mentioned, based on the search algorithm, similarity measures are selected from the evaluation data for the whole data set. However, the algorithm is flexible so that, in different conditions, different classifiers can be adopted adaptively. For example considering the SFS algorithm, in the case of the G protocol the GD, Chebyschev, Chi-square $\left(\chi^{2}\right)$,Canberra and Manhattan distances were selected for manually registered data, whereas the GD, NC and Correlation Coefficient metrics were adopted in the Ua protocol. A comparison of the SFS and $+2-1$ results demonstrate that in most of the cases a similar performance is obtained using these algorithms. Our investigations confirm that a same subset of the scoring functions is selected using both methods when a same performance is achieved. As the result, since the SFS method is simpler than $+2-1$ method, applying this algorithm is sufficient in order to find the optimum metrics.

Overall, these results clearly demonstrate that the proposed similarity measures selection and fusion algorithms considerably improves the performance of the face verification system. The only exception is for the manually registered data of the MA protocol where the GD metric individually outperforms the combined system. It should be noted that in these experiments our thresholding technique has been applied for decision making when a metric is used individually while the SVM classifier has been used in the selection and fusion procedure.

\section{Conclusions}

We addressed the problem of selecting and fusing similarity measure based classifiers for face authentication in the LDA face space. An SVM-based sequential search approach similar to the "plus L, and take away R" algorithm was applied in order to find an optimum subset of similarity measures to be fused as a basis for decision making. Using the proposed method, the performance of the verification system can be considerably improved. 
Acknowledgements. The financial support from Iran Telecommunication Research Centre is gratefully acknowledged. A partial support from the EU Network of Excellence Biosecure is also gratefully acknowledged.

\section{References}

1. Belhumeur, P.N., Hespanha, J.P., Kriegman, D.J.: Eigenfaces vs. Fisherfaces: Recognition using class specific linear projection. IEEE Trans. on Pattern Recognition and Machine Intelligence 19(7), 711-720 (1997)

2. Devijver, P., Kittler, J.: Pattern Recognition: A Statistical Approach. PrenticeHall, Englewood Cliffs (1982)

3. Duin, R.P.W.: The combining classifier: to train or not to train? In: Inter. Conf. on Pattern Recognition (2002)

4. Freund, Y., Schapire, R.E.: A decision-theoretic generalization of on-line learning and an application to boosting. Journal of Computer and System Sciences 55 (August 1997)

5. Hamouz, M., Kittler, J., Kamarainen, J.-K., Paalanen, P., Kalviainen, H., Matas, J.: Feature-based affine-invariant localization of faces. IEEE Transactions on Pattern Analysis and Machine Intelligence 27(9), 1490-1495 (2005)

6. Kittler, J., Hatef, M., Duin, R.P.W., Matas, J.: On combining classifiers. IEEE Trans. on Pattern Recognition and Machine Intelligence, PAMI 20(3), 226-239 (1998)

7. Kittler, J., Roli, F.: Multiple classifier systems, vol. 2096. Springer, Heidelberg (2001)

8. Maghooli, K., Moin, M.S.: A new approach on multimodal biometrics based on combining neural networks using adaboost. In: ECCV Workshop BioAW (2004)

9. Perlibakas, V.: Distance measures for pca based face recognition. Pattern Recognition letters 25(6) (2004)

10. Pudil, P., Novovicova, J., Kittler, J.: Floating search methods in feature selection. Pattern Recognition Letters 15, 1119-1125 (1994)

11. Roli, F., Fumera, G.: Analysis of linear and order statistics combiners for fusion of imbalanced classifiers. In: 3rd Int. Workshop on Multiple Classifier Systems, Cagliari, Italy. Springer, Heidelberg (2002)

12. Sadeghi, M., Kittler, J.: Decision making in the LDA space: Generalised gradient direction metric. In: the 6th International Conference on Automatic Face and Gesture Recognition, Seoul, Korea, pp. 248-253 (May 2004)

13. Sadeghi, M., Kittler, J.: Confidence based gating of multiple face authentication experts. In: Joint IAPR International Workshops on Syntactical and Structural Pattern Recognition and Statistical Pattern Recognition, S+SSPR 2006, Hong Kong, China, August 17-19, pp. 667-676 (2006)

14. Sadeghi, M., Samiei, M., Kittler, J., Almodarresi, M.: Similarity measures fusion using svm classifier for face authentication. In: Proceedings of the third International Conference on Computer Vision Theory and Applications, VISAPP 2008, Portugal, vol. 2, pp. 105-110 (January 2008)

15. Vapnik, V.: The Nature of Statistical Learning Theory. Springer, New York (1995)

16. Verikas, A., Lipnickas, A., Malmqvist, K., Bacauskiene, M., Gelzinis, A.: Soft combining of neural classifiers: A comparative study. Pattern Recognition Letters 20, 429-444 (1999)

17. Xu, L., Krzyzak, A., Suen, C.Y.: Methods of combining multiple classifiers and their applications to handwriting recognition. IEEE Trans. on Systems, Man, and Cybernetics 22, 418-435 (1992) 